\title{
Upper Nasopharyngeal Corridor for Transnasal Endoscopic Drainage of Petroclival Cholesterol Granulomas: Alternative Access in Conchal Sphenoid Patients
}

\author{
Nefize Turan ${ }^{1}$ Griffin R. Baum ${ }^{1} \quad$ Christopher M. Holland ${ }^{1} \quad$ Faiz U. Ahmad ${ }^{1}$ Oswaldo A. Henriquez ${ }^{2}$ \\ Gustavo Pradilla ${ }^{1}$ \\ ${ }^{1}$ Emory University School of Medicine, Department of Neurosurgery, \\ Atlanta, GA, United States \\ 2 Emory University School of Medicine, Department of \\ Otolaryngology, Head and Neck Surgery, Atlanta, GA, United States \\ Address for correspondence Gustavo Pradilla, MD, Department of \\ Neurosurgery, Emory University School of Medicine, 1365 Clifton Rd. \\ NE, Suite B6166, Atlanta, GA, United States 30322 \\ (e-mail: gpradil@emory.edu).
}

J Neurol Surg Rep 2016;77:e17-e22.

\begin{abstract}
Keywords

- cholesterol granuloma

- endoscopic

- nasopharyngeal

- petroclival

- petrous apex

Background Cholesterol granulomas arising at the petrous apex can be treated via traditional open surgical, endoscopic, and endoscopic-assisted approaches. Endoscopic approaches require access to the sphenoid sinus, which is technically challenging in patients with conchal sphenoidal anatomy.

Clinical Presentation A 55-year-old woman presented with intermittent headaches and tinnitus. Formal audiometry demonstrated moderately severe bilateral hearing loss. $\mathrm{CT}$ of the temporal bones and sella revealed a well-demarcated expansile lytic mass. MRI of the face, orbit, and neck showed a right petrous apex mass measuring $22 \times 18$ $\times 19 \mathrm{~mm}$ that was hyperintense on T1- and T2-weighted images without enhancement, consistent with a cholesterol granuloma. The patient had a conchal sphenoidal anatomy.

Operative Technique Herein, we present an illustrative case of a low-lying petroclival cholesterol granuloma in a patient with conchal sphenoidal anatomy to describe an alternative high nasopharyngeal corridor for endoscopic transnasal transclival access. Postoperative Course Postoperatively, the patient's symptoms recovered and no complications occurred. Follow-up imaging demonstrated a patent drainage tract without evidence of recurrence.

Conclusion In patients with a conchal sphenoid sinus, endoscopic transnasal transclival access can be gained using a high nasopharyngeal approach. This corridor facilitates safe access to these lesions and others in this location.
\end{abstract}

\section{Background}

Cholesterol granulomas (CGs) are benign lesions of the temporal bone that develop most frequently at the petrous apex

Content of this work was presented in poster presentation form at the 25th Annual North American Skull Base Society Meeting Tampa, Florida, USA, February 20-22, 2015.

received

October 5, 2015

accepted

September 14, 2015

published online

November 16, 2015
(PA). ${ }^{1}$ These granulomatous lesions are cystic with a fibrous capsule and are often filled with a brown or yellow fluid. Extensive pneumatization of the PA has been identified as a major risk factor for CG formation and was observed in 10 to $30 \%$ of patients with CG. CGs are the most common primary lesions in this location ${ }^{2}$ but can also be found in the mastoid bone, middle ear cavity, paranasal sinuses, orbitofrontal bone,
License terms

Stuttgart - New York

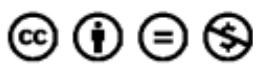


and in the petroclival region., ${ }^{3,4}$ They are distinct from cholesteatomas, which are also found in many of the same regions.

The pathogenesis of CGs has traditionally been explained by two theories: the obstructive-vacuum and the exposed marrow hypothesis. The former and more classic concept states that mucosal edema causes obstruction of air cell tracts and creates a vacuum effect resulting in extravasation of blood into these spaces and subsequent granulomatous reaction to cholesterol and blood products, forming the pathognomonic lesion, which grossly appears as an intraosseous cyst filled with dark, viscous, chocolate brown fluid and granulation tissue. ${ }^{2}$ Conversely, the exposed marrow hypothesis postulates that hyperpneumatization of the PA predisposes to bone marrow-air cell interface formation and bleeding from hypervascular bone marrow blebs. ${ }^{5-7}$

Clinically these lesions are benign and often remain asymptomatic until significant mass effect occurs leading to cranial nerve dysfunction. However, most commonly, headache is the presenting symptom ${ }^{8,9}$ followed by hearing loss, vestibular dysfunction, tinnitus, diplopia, and ipsilateral retro-orbital pain. $^{2}$ On radiologic imaging, CGs appear characteristically as expansile and erosive cystic lesions with well-defined margins on computed tomography (CT) and as high-signal intensity lesions on both T1- and T2-weighted images on magnetic resonance imaging (MRI) without contrast enhancement. ${ }^{2}$ These lesions have a similar appearance to cholesteatomas on CT; however, CGs are hyperintense on both T1- and T2-weighted imaging and cholesteatomas are $\mathrm{T} 1$ hypointense.

Traditionally CGs were treated surgically with open transcranial approaches, including infralabyrinthine, transcanal infracochlear, middle cranial fossa, transotic, translabyrinthine, and suboccipital retrosigmoid. ${ }^{1,10}$ The surgical exposure of the petroclival region necessary to access CGs, however, can be challenging due to the morphology of the petrous bone and the neurovascular contents of the peripetrous complex. ${ }^{11}$ Endoscopic and endoscopic-assisted endonasal approaches are increasingly used as less invasive alternatives that allow wide panoramic exposure and extended visualization. ${ }^{10}$

Endonasal access is gained most commonly through a transsphenoidal corridor, which requires favorable sphenoidal anatomy. Four types of sphenoid sinus pneumatization have been described in the literature: conchal (nonpneumatized) $(2 \%)$, presellar (21\%), sellar (54.7\%), and postsellar $(22.3 \%) .^{12}$ In patients with a conchal sphenoid, a transsphenoidal approach is technically difficult. In this report, we present a case of a low-lying petroclival CG in a patient with conchal sphenoidal anatomy treated with an endoscopic transnasal transclival approach via a high nasopharyngeal corridor that illustrates the technique for nasopharyngeal access to these lesions.

\section{Clinical Presentation}

A 55-year-old woman presented with intermittent headaches and tinnitus without other neurological symptoms. Physical examination was unremarkable and formal audiometry demonstrated moderately severe bilateral hearing loss. CT of the temporal bones and sella revealed a well-demarcated expansile lytic mass with soft tissue density, centered in the right PA, with medial extension into the clivus and petroclival synchondrosis, inferior extension into the carotid space, and cephalic extension to the right petrous ridge (-Fig. $1 \mathrm{~A}-\mathbf{C}$ ). Medially, the mass scalloped the adjacent clivus but did not extend into the sphenoid sinus. Marked thinning of the medial wall of the vertical petrous segment and the posterior wall of the horizontal petrous ICA canals with large areas of complete bone density loss were concerning for extreme thinning versus dehiscence. The horizontal petrous ICA was also displaced slightly anteriorly by the soft tissue mass. Conchal anatomy of the sphenoid sinus was also noted. MRI of the face, orbit, and neck showed a right PA mass measuring $22 \times 18 \times 19 \mathrm{~mm}$ that was hyperintense on T1- and T2-weighted images without enhancement, consistent with CG ( - Fig. 1 D-F). Given her symptomatic presentation treatment was recommended and in light of her sphenoidal anatomy, an upper nasopharyngeal corridor was selected to perform a transclival endoscopic approach to the lesion.

\section{Operative Technique}

Preoperative imaging studies including maxillofacial and sinus CT as well as a craniofacial MRI were acquired with a standard high-resolution protocol and loaded into a neuronavigation system (Medtronic S7 StealthStation, Louisville, Colorado, United States). Three-dimensional images were reconstructed and the patient underwent co-registration of the stereotactic images with surface landmarks. Registration accuracy was verified using known anatomic landmarks and the scalp surface contour. A right maxillary antrostomy was performed, and a pedicled nasoseptal flap was elevated. Middle turbinectomy was performed to maximize the exposure and the natural antrum was identified and opened. Following identification of the nasopharyngeal and eustachian tube landmarks, the mucosae of the nasopharyngeal area and adenoid bed were completely removed using coblation (ArthroCare ENT, Austin, Texas, United States) (-Fig. 2 A-C). After this exposure, the right vidian canal and nerve as well as the sphenopalatine canal were identified on the right side. Using a high-speed drill with a diamond bur (Medtronic Visao High-Speed Otologic Drill, Minneapolis, Minneapolis, United States) and neuronavigation, the transclival exposure was performed from anterior to posterior and from medial to lateral. An endoscopic Doppler probe (Mizuho $8 \mathrm{MHz}$ Surgical Doppler System, Union City, California, United States) was used throughout the procedure to identify the course of the internal carotid artery (ICA) at its transition from cervical to paraclival above the opening of the eustachian tube. Using cottle elevators and angled dissectors, the plane between the clivus and the paraclival soft tissues was identified, and drilling proceeded posteriorly and laterally until the cyst contents were reached. Inside the cystic cavity, membranes and brown/yellow material with an oil-like fluid 

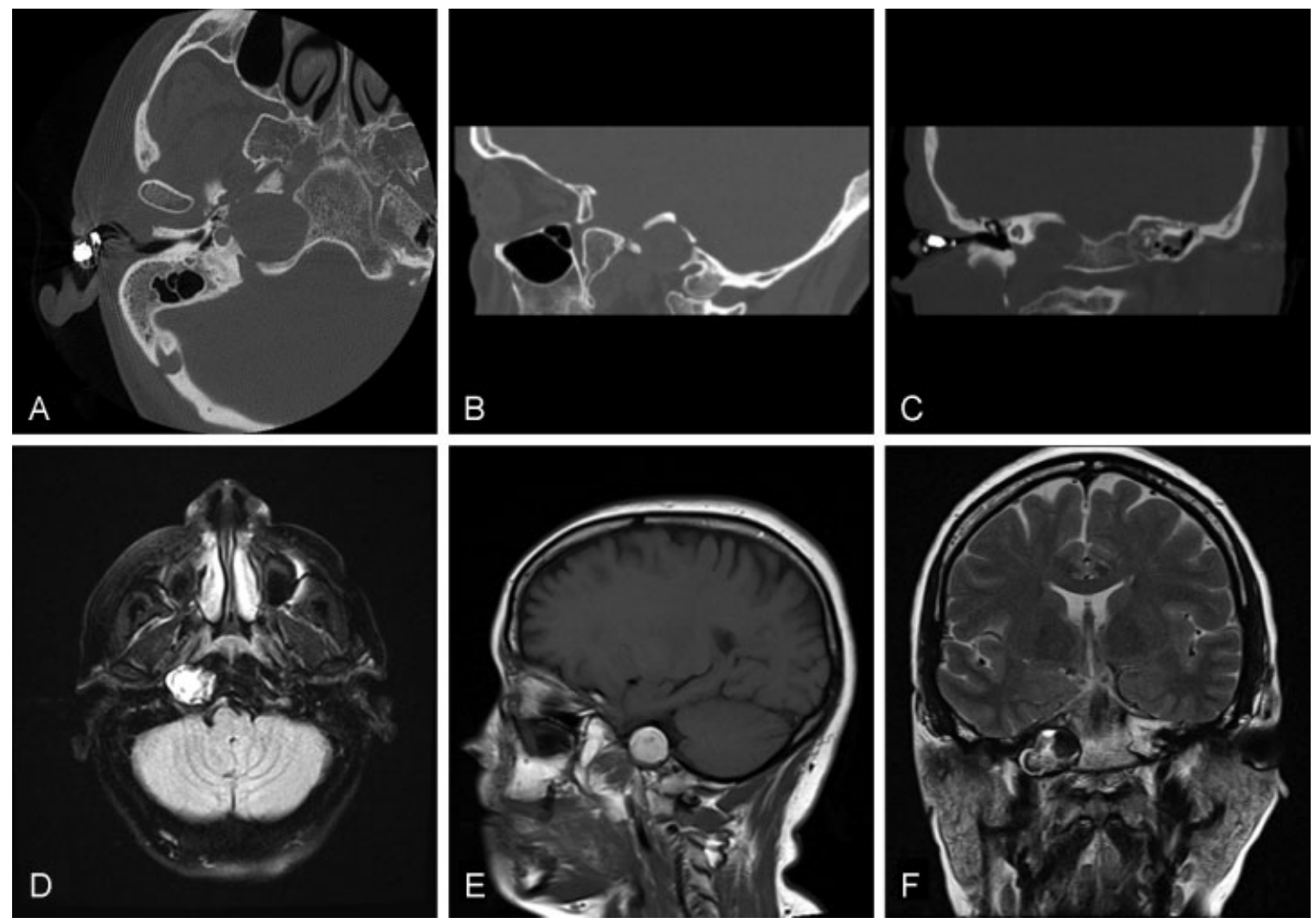

Fig. 1 Preoperative axial, sagittal, and coronal CT images demonstrating a lytic expansile soft tissue mass within the PA (A-C). Preoperative MRI (D-F) demonstrates a lesion with hyperintensity on both T1- and T2-weighted imaging consistent with a cholesterol granuloma (CG).

were encountered, and these were evacuated using a combination of 45- and 90-degree pituitary curettes as well as forceful irrigation with saline, and a combination of straight and angled suctions under direct visualization with the 0 degree endoscope. Once all the cyst contents were clearly evacuated, a 30-degree endoscope was advanced to inspect the cystic cavity and complete the decompression ( - Fig. 2 DJ). Using the NICO Myriad aspirator (NICO Corporation, Indianapolis, Indiana, United States) additional areas of the cyst wall were resected and the diamond bur was used to extend drilling medially, superiorly, and inferiorly enlarging the caliber of the outlet tract to facilitate placement of the nasoseptal flap and tract mucosalization. Hemostasis was achieved and the nasoseptal flap was set in place along the decompression tract ( $\mathbf{F i g .} \mathbf{2} \mathbf{K}-\mathbf{0}$ ).

\section{Postoperative Course}

The patient's postoperative course was unremarkable, and the postoperative MRI and CT demonstrated effective evacuation of the cyst contents and an open drainage tract from the resection cavity (-Fig. 3 A-D). She was discharged home in good condition on postoperative day 3 . Tinnitus and headaches improved and outpatient follow-up evaluations were conducted at 1,3, and 9 months postop. Endoscopic outpatient examination at 1 month revealed patency of the outflow tract. A subsequent endoscopic examination at 3 months showed partial stenosis of the outflow tract without recur- rence of the lesion. Follow-up CT obtained 9 months after surgery showed a stable outflow tract without evidence of recurrence. Audiogram obtained at 9 months showed stable mild to moderate sensorineural hearing loss with 90 to $100 \%$ speech recognition preservation.

\section{Discussion}

The endoscopic transsphenoidal approach to petrous apex cholesterol granulomas (PACGs) was first described by Fucci et al in $1994 .{ }^{13}$ Subsequently, Mattox suggested using the endoscope as an adjunct during open surgical approaches and reported that the endoscopic tools were useful to mobilize and remove debris within long-standing CGs, remove septations, and drain multiloculated cysts. ${ }^{14}$ The addition of image guidance and improvements in endoscopic visualization and instrumentation enabled the widespread use of endoscopic transsphenoidal approaches, especially in patients with lesions neighboring the posterior wall of the sphenoid sinus. ${ }^{15-20}$

Hearing preservation and permanent aeration of the cyst cavity constitute the main goals of treatment for CGs. Cyst drainage is therefore sufficient and complete removal of the cyst wall is not required. ${ }^{21}$ Scopel and colleagues have shown that endonasal approaches can provide drainage windows three times larger than those achieved with transcanal infracochlear approaches, and therefore may be associated with better outcomes and decreased recurrence. ${ }^{22} \mathrm{~A}$ recent 


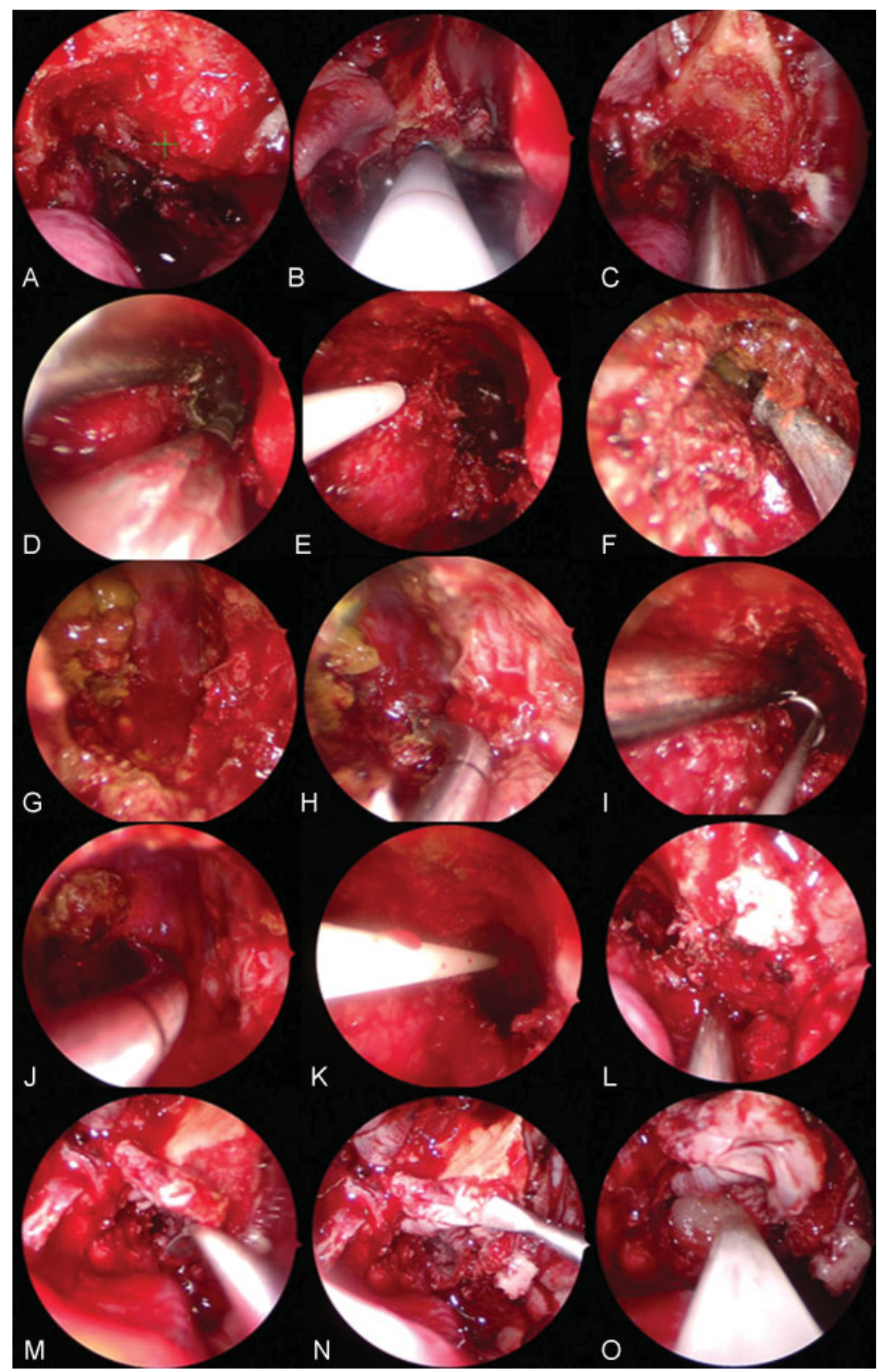

Fig. 2 Intraoperative images. (A-C) The upper nasopharyngeal corridor used for access. (D-J) Sequential cyst decompression. (K-O) Nasoseptal flap positioning.

systematic review by Eytan et al included 53 patients with PACGs from 22 studies and showed a $20 \%$ restenosis rate and $7.5 \%$ overall recurrence after endoscopic treatment. ${ }^{23} \mathrm{Com}-$ pared with $12.5 \%$ overall recurrence rate in open surgical approaches reported in the same study, the clinical outcomes of endoscopically managed CGs are thought to be favorable although prospective studies are needed to confirm these results. ${ }^{23}$ Although miniflaps and stents have been used to decrease the recurrence of CGs8, Eytan et al did not find significant decrease in recurrence rates with stenting. ${ }^{23}$

Surgical decision making and selection of the appropriate approach is dependent on hearing status of the patient at 

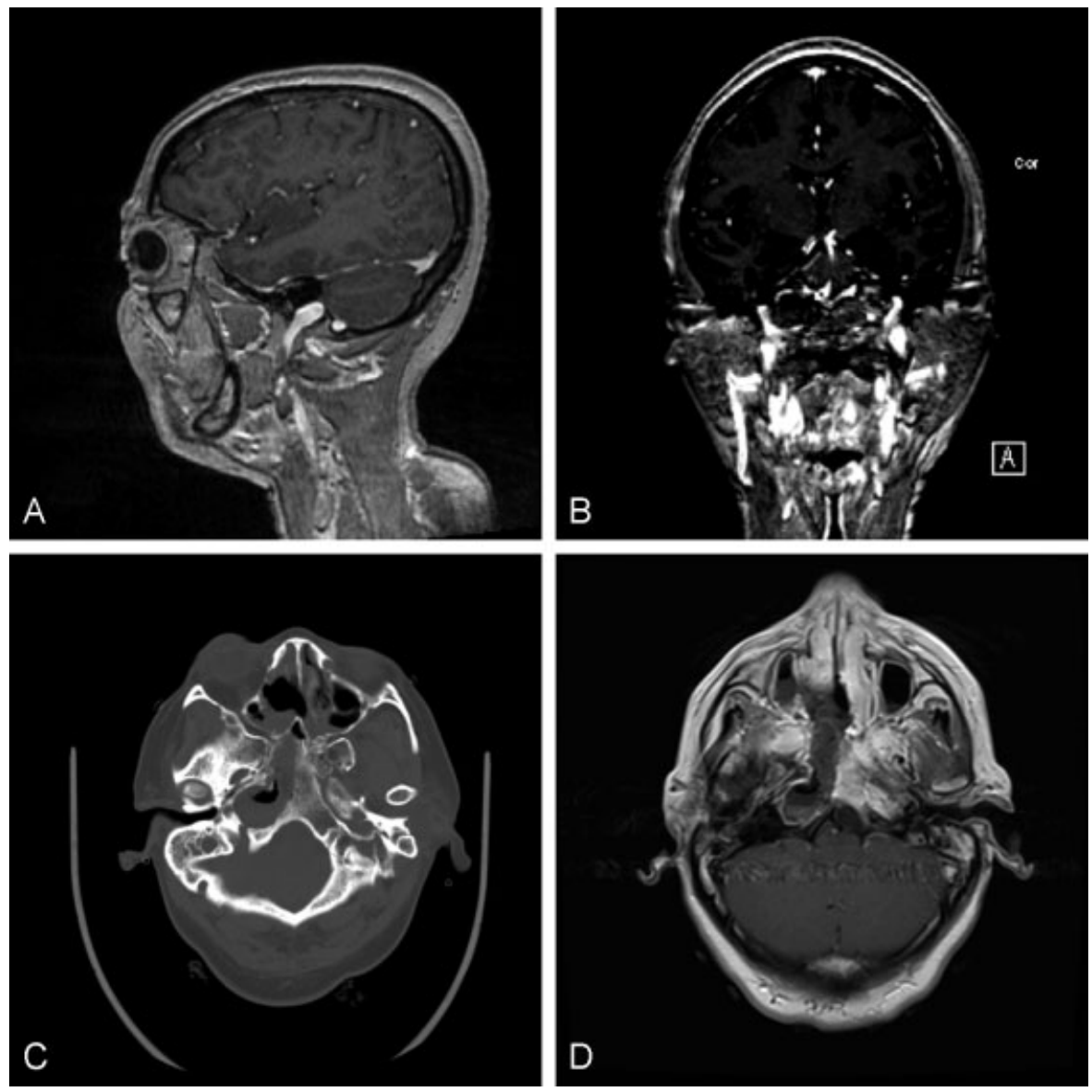

Fig. 3 Postoperative MRI (A, B, and D) and CT (C) demonstrate effective drainage with ample evacuation of the cyst contents and an open drainage tract from the resection cavity.

presentation, pneumatization of the mastoid cavity and PA, location of the ICA in relation to the lesion, size and pneumatization of the sphenoid sinus, and ability to achieve permanent drainage. ${ }^{13}$ In particular, as the anatomic variations and the pattern of pneumatization in the sphenoid sinus can have a direct effect on surgical planning, careful preoperative evaluation of the anatomy of the sphenoid sinus by CT scan and MRI are of paramount importance. Endoscopic approaches such as the medial transsphenoidal approach with ICA lateralization and the transpterygoid infrapetrous approach have been described for the treatment of CGs. ${ }^{10}$ The medial transsphenoidal approach involves removal of the posterior septum and rostrum with wide sphenoidotomies to provide access to the lateral sphenoid. ${ }^{24}$ When the lesion is poorly visualized due to poor pneumatization of the sphenoid sinus, posterolateral localization, or lack of medial expansion of the lesion, a medial approach can be performed with additional bone removal and ICA lateralization. ${ }^{24}$ In that case, the ICA can be decompressed and lateralized using the vidian nerve and artery as landmarks to identify the paraclival ICA. In those cases, the clivus can be further drilled until the clival dura is exposed for augmentation of the surgical corridor. These approaches, however, rely on favorable sphenoidal anatomy for access.

When the sphenoid is not accessible due to poor pneumatization, a transpterygoid infrapetrous approach has also been described. ${ }^{24}$ This approach involves eustachian tube and foramen lacerum dissection, vidian artery and nerve exposure and mobilization, drilling of the pterygoid plates, and access through the eustachian tube. The PA is reached inferiorly by drilling the bone between the horizontal segment of the petrous ICA and the eustachian tube. ${ }^{24}$ Once the horizontal and vertical segments of the ICA are outlined, drilling is performed below the petrous ICA until the lesion is accessed. ${ }^{24}$ Compared with this approach, the operative technique in the present report describes an alternative transclival corridor using a high nasopharyngeal trajectory with intraoperative image and Doppler guidance for ICA localization with the help of anatomic landmarks such as the vidian nerve and artery. This alternative approach can be implemented patients with conchal sphenoidal anatomy.

\section{Conclusion}

Conchal sphenoidal anatomy, although rare, occurs in up to $2 \%$ of patients. ${ }^{12}$ Access to the PA via endoscopic transsphenoidal approaches can be difficult in patients with a poorly pneumatized sphenoid as in the illustrative case reported. Therefore, use of a high nasopharyngeal corridor in conjunction with intraoperative navigation, Doppler ultrasonography, and external landmarks such as the vidian canal provides broad access to the PA for effective drainage of these lesions. ${ }^{8}$ 
e22 Endoscopic Drainage of CGs in Conchal Sphenoids Turan et al.

\section{Sources of Financial and Material Support}

None.

\section{References}

1 Brackmann DE, Toh EH. Surgical management of petrous apex cholesterol granulomas. Otol Neurotol 2002;23(4):529-533

2 Isaacson B, Kutz JW, Roland PS. Lesions of the petrous apex: diagnosis and management. Otolaryngol Clin North Am 2007; 40(3):479-519, viii

3 Durgam A, Batra PS. Paranasal sinus cholesterol granuloma: systematic review of diagnostic and management aspects. Int Forum Allergy Rhinol 2013;3(3):242-247

4 Imre A, Pinar E, Paker I, Imre SS, Duran R, Ozturkcan S. Osteoplastic flap approach versus orbitotomy in case of orbitofrontal cholesterol granuloma. J Craniofac Surg 2015;26(1):e36-e37

5 Jackler RK, Cho M. A new theory to explain the genesis of petrous apex cholesterol granuloma. Otol Neurotol 2003;24(1):96-106, discussion 106

6 Selman Y, Wood JW, Telischi FF, Casiano RR, Angeli SI. Development of cholesterol granuloma in a temporal bone petrous apex previously containing marrow exposed to air cells. Otol Neurotol 2013;34(5):958-960

7 Hoa M, House JW, Linthicum FH Jr. Petrous apex cholesterol granuloma: maintenance of drainage pathway, the histopathology of surgical management and histopathologic evidence for the exposed marrow theory. Otol Neurotol 2012;33(6):1059-1065

8 Paluzzi A, Gardner P, Fernandez-Miranda JC, et al. Endoscopic endonasal approach to cholesterol granulomas of the petrous apex: a series of 17 patients: clinical article. J Neurosurg 2012; 116(4):792-798

9 Sade B, Batra PS, Scharpf J, Citardi MJ, Lee JH. Minimally invasive endoscopic endonasal management of skull base cholesterol granulomas. World Neurosurg 2012;78(6):683-688

10 Gore MR, Zanation AM, Ebert CS, Senior BA. Cholesterol granuloma of the petrous apex. Otolaryngol Clin North Am 2011;44(5): 1043-1058

11 Fournier HD, Mercier P, Roche PH. Surgical anatomy of the petrous apex and petroclival region. Adv Tech Stand Neurosurg 2007; 32:91-146
12 Hamid O, El Fiky L, Hassan O, Kotb A, El Fiky S. Anatomic variations of the sphenoid sinus and their impact on trans-sphenoid pituitary surgery. Skull Base 2008;18(1):9-15

13 Fucci MJ, Alford EL, Lowry LD, Keane WM, Sataloff RT. Endoscopic management of a giant cholesterol cyst of the petrous apex. Skull Base Surg 1994;4(1):52-58

14 Mattox DE. Endoscopy-assisted surgery of the petrous apex. Otolaryngol Head Neck Surg 2004;130(2):229-241

15 DiNardo LJ, Pippin GW, Sismanis A. Image-guided endoscopic transsphenoidal drainage of select petrous apex cholesterol granulomas. Otol Neurotol 2003;24(6):939-941

16 Presutti L, Villari D, Marchioni D. Petrous apex cholesterol granuloma: transsphenoid endoscopic approach. J Laryngol Otol 2006; 120(6):e20

17 Prabhu K, Kurien M, Chacko AG. Endoscopic transsphenoidal approach to petrous apex cholesterol granulomas. $\mathrm{Br} \mathrm{J}$ Neurosurg 2010;24(6):688-691

18 Dhanasekar G, Jones NS. Endoscopic trans-sphenoidal removal of cholesterol granuloma of the petrous apex: case report and literature review. J Laryngol Otol 2011;125(2):169-172

19 Jaberoo MC, Hassan A, Pulido MA, Saleh HA. Endoscopic endonasal approaches to management of cholesterol granuloma of the petrous apex. Skull Base 2010;20(5):375-379

20 Chatrath P, Nouraei SA, De Cordova J, Patel M, Saleh HA. Endonasal endoscopic approach to the petrous apex: an image-guided quantitative anatomical study. Clin Otolaryngol 2007;32(4):255-260

21 Gherini SG, Brackmann DE, Lo WW, Solti-Bohman LG. Cholesterol granuloma of the petrous apex. Laryngoscope 1985;95(6): 659-664

22 Scopel TF, Fernandez-Miranda JC, Pinheiro-Neto CD, et al. Petrous apex cholesterol granulomas: endonasal versus infracochlear approach. Laryngoscope 2012;122(4):751-761

23 Eytan DF, Kshettry VR, Sindwani R, Woodard TD, Recinos PF. Surgical outcomes after endoscopic management of cholesterol granulomas of the petrous apex: a systematic review. Neurosurg Focus 2014;37(4):E14

24 Zanation AM, Snyderman CH, Carrau RL, Gardner PA, Prevedello DM, Kassam AB. Endoscopic endonasal surgery for petrous apex lesions. Laryngoscope 2009;119(1):19-25 